\title{
Design of Physics Experiment Based on Uncertainty Theory
}

\author{
HuiWen Liu
}

\author{
Jingdezhen Ceramic Institute, Jingdezhen, China, 333403
}

\section{Keywords: Uncertainty theory; Experimental theory; Experimental scheme; Experimental design}

\begin{abstract}
This paper is based on the theory of uncertainty, will choose the smallest uncertainty as the ultimate goal, and based on that to choose the optimal experimental theory and experimental program. This study can be designed to experiment, in order to screen out the most appropriate program, which play a role in guiding the theory. This article makes detailed analysis by combining with the practical case.

Measurement is an indispensable task for physical experiments, but in the measurement process, the errors are almost inevitable. In the nineties of last century, International Bureau of Metrology specially developed a corresponding guide, China has also developed a measurement error and uncertainty for the relevant guidelines, the guidelines clearly indicate the accuracy of the measurement results should be expressed by using "uncertainty", the education headquarters also made a request for the university's physical experiment, that is, to establish the "experimental evaluation system" of the uncertainty, fully applied to the university's physical experiments. In the study of physical experiments, physical experiment design is one of the very important contents, especially because of the continuous progress of science and technology in recent years, the requirements of talent is also getting higher and higher, so in the study of university physics experiments, innovative experiment research has become increasingly important. Which is carried out mainly through a reasonable and feasible experimental design, and ultimately not only continuously enhance the students' innovative consciousness and innovative thinking, but also make the measurement results more accurate, so as to provide real and reliable measurement data.
\end{abstract}

\section{The Theory of Uncertainty}

With regard to the measurement of uncertainty, which is composed of many components together, through the classification of these components, they can be divided into two categories: the first category components of the assessment is calculated through the statistical data to achieve; The evaluation of the second category component is achieved by empirical or other probabilistic distributions of data information, and the representation is the standard deviation. Because of the different methods of assessment, the uncertainty can be divided into two types, the uncertainty assessment method for A class is statistics, and uncertainty assessment methods for B class are other methods except for statistics. In the following, two types of uncertainty will be described in detail by the formula.

The evaluation formula of uncertainty for class $\mathrm{A}$ is:

$$
u_{A}=S(\bar{x})=\sqrt{\sum_{\mathrm{i}=1}^{\mathrm{n}}\left(x_{i}-\bar{x}\right)^{2} / \mathrm{n}(\mathrm{n}-1)}
$$

In the above formula, $x_{i}$ represents the measure value of repeated measurements to $\mathrm{i} ; \bar{x}$ represents the average of the $\mathrm{n}$ times measurements. Where $\mathrm{n}$ is the number of repetitions; $u_{A}$ refers to uncertainty of Class A.

The evaluation formula of uncertainty for class B is:

$$
u_{B}=\Delta / K_{p}
$$

In this formula, $\Delta$ represents the instrument's limit error; $u_{B}$ refers to uncertainty of the class B. The value of $K_{p}$ is determined by the probability distribution of the error. When the normal distribution is given, the value of $K_{p}$ is 3 ; when it is evenly distributed, the value of $K_{p}$ is $\sqrt{3}$; when it is the triangular distribution, the value of $K_{p}$ is $\sqrt{6}$.

Uncertainty of univariate measurement is: 
$u_{c}=\sqrt{u_{B}^{2}+u_{A}^{2}}$

In this formula, $u_{B}$ and $u_{A}$ the two uncertainties are independent of each other.

Formula of indirect measurement of uncertainty is:

$u\left(x_{i}\right)=\sqrt{u_{B}^{2}\left(x_{i}\right)+u_{A}^{2}\left(x_{i}\right)}$

From the above formula, the final synthetic standard uncertainty formula we can get is:

$$
u_{c}^{2}(y)=\sum_{\mathrm{i}=1}^{\mathrm{n}}\left(\frac{\partial f}{\partial x_{i}}\right)^{2} u_{A}^{2}\left(x_{i}\right)+\sum_{\mathrm{i}=1}^{\mathrm{n}}\left(\frac{\partial f}{\partial x_{i}}\right)^{2} u_{B}^{2}\left(x_{i}\right)
$$

\section{The Choice of the Best Experimental Program}

Through the physical experiment design, it can effectively improve the students' practical operation ability and innovation ability. According to the specific requirements of the experiment, selecting the appropriate experimental theory and experimental measurement method is the experimental design. A specific case will be combined with in the following, using uncertainty theory to select the optimal experimental program, so that the experiment can be more reasonable and feasible, but also the uncertainty can be controlled in the minimum range.

\section{The Choice of Experimental Theory}

In the course of the experiment, the measurement methods for indirect measurement object are usually very rich, but for the measurement results, it is natural to try to minimize the value of uncertainty, so the choice of experimental theory, it can be set to the same accuracy and then compare the value of the uncertainty, in this way, you can choose the most suitable experimental theory.

In the following, we will give a detailed description of the volume of the cylinder. We can denote the outer diameter of the cylinder by $d_{1}$, the inner diameter is denoted by $d_{2}$ and the distance from the inner edge of the cylinder to the outer cylinder is denoted by $d$, The height of cylinder is denoted by $h$.

The formula for measuring the cylinder volume is:

$$
\begin{aligned}
& V_{1}=\frac{\pi}{4}\left(d_{1}{ }^{2}-d_{2}{ }^{2}\right) h \\
& V_{2}=\pi\left(d_{1}-\mathrm{d}\right) \mathrm{d} h
\end{aligned}
$$

Since the measurement tool used is the same vernier caliper when measuring the values of $d_{1}, d_{2}$, and $d$, the class B uncertainty should be used in the course of this experimental design, and $\mathrm{ud}_{1}=\mathrm{ud}_{2}=\mathrm{u}_{\mathrm{d}}$. Combined with the formula to calculate, the uncertainty of $V_{1}$ and $V_{2}$ we can get are:

$$
\begin{aligned}
& \frac{\mathrm{u}\left(V_{1}\right)}{V_{1}}=\sqrt{4 \frac{{d_{1}}^{2}+d_{2}{ }^{2}}{\left(d_{1}{ }^{2}-d_{2}{ }^{2}\right)^{2}} u_{d}{ }^{2}+\frac{u_{A}{ }^{2}}{h^{2}}} \\
& \frac{\mathrm{u}\left(V_{2}\right)}{V_{2}}=\sqrt{\frac{5 d_{1}{ }^{2}+d_{2}{ }^{2}-4 d_{1} \mathrm{~d}}{\left(\mathrm{~d}_{1}-d_{2}\right)^{2} \mathrm{~d}^{2}} u_{d}{ }^{2}+\frac{u_{A}{ }^{2}}{h^{2}}}
\end{aligned}
$$

From the above two formulas we can calculate the average difference of uncertainty between the two methods, because $d_{2}=d_{1}-2 d$, we can draw the following formula:

$$
\left(\frac{\mathrm{u}\left(V_{1}\right)}{V_{1}}\right)^{2}-\left(\frac{\mathrm{u}\left(V_{2}\right)}{V_{2}}\right)^{2}=\left[\left(\frac{\mathrm{u}\left(V_{1}\right)}{V_{1}}\right)+\left(\frac{\mathrm{u}\left(V_{2}\right)}{V_{2}}\right)\right] \cdot\left[\left(\frac{\mathrm{u}\left(V_{1}\right)}{V_{1}}\right)+\left(\frac{\mathrm{u}\left(V_{2}\right)}{V_{2}}\right)\right]=\frac{2 d_{1}-3 d}{d\left(d_{1}-d\right)^{2}} u_{d}{ }^{2}
$$

By analyzing the above formula, we can know that if the value is greater than 1.5 times of $\mathrm{d}$, the uncertainty obtained from using the first method is higher than the second method, that is, the results use the second method to get are more consistent with the real situation, then, the optimal measurement theory is formula(8). If the value is less than 1.5 times of $\mathrm{d}$, then the $\frac{\mathrm{u}\left(V_{2}\right)}{V_{2}}-\frac{\mathrm{u}\left(V_{1}\right)}{V_{1}}$. is greater than zero. In this case, the uncertainty of the method 1 used is less than that of the method 2. In this case, the optimal measurement theory is formula (7). If the value is equal to 1.5 times of $d$, 
then the $\frac{\mathrm{u}\left(V_{2}\right)}{V_{2}}-\frac{\mathrm{u}\left(V_{1}\right)}{V_{1}}$ is zero, in which case, the uncertainty obtained from two methods are the same, either one of them can be selected.

\section{Selection of Measurement Scheme}

After a clear understanding of the experimental theory to be used, it is necessary to select the measurement scheme, because it also plays a very important role in the whole experimental design. In the experimental design, if you want to measure the value of a certain of indirect parameter, usually first of all you need to measure several direct parameters, and ultimately to achieve the purpose of measuring the indirect parameter. The accuracy of the measurement results has a large relationship with the specific range of the measurement object. In the following an example was used to carry out specific analysis, the selected example is through the free-fall movement to measure the acceleration of gravity, mainly by measuring the scope of the object by how the uncertainty of the impact to get the minimum uncertainty, and finally get the most suitable measurement program. This example is: from top to bottom, the position of the electro-optical door is divided into E0, E1, E2, the distance between E0 and E1 is S1, the time used is t1, the distance between E0 and E2 is $\mathrm{S} 2$, time is $\mathrm{t}$ 2, the value of gravity acceleration $\mathrm{g}$ can be obtained by the following formula.

$$
\mathrm{S}=V_{0} \mathrm{t}+1 / 2 \mathrm{gt}^{2}
$$

So the value of $\mathrm{g}$ we can get is:

$$
g=2 \frac{\frac{s_{2}}{t_{2}}-\frac{s_{1}}{t_{1}}}{t_{2}-t_{1}}=2 s_{2} t_{1}-s_{1} t_{2} / t_{1} t_{2}\left(t_{2}-t_{1}\right)
$$

Through this formula, the square of the uncertainty of $g$ we can get is:

$$
\left[\frac{\mathrm{u}(\mathrm{g})}{\mathrm{g}}\right]^{2}=\left\{\left(\frac{\partial \mathrm{g}}{\partial \mathrm{s}_{2}} \mathrm{u}_{\left(\mathrm{s}_{2}\right)}\right)^{2}+\left(\frac{\partial \mathrm{g}}{\partial \mathrm{s}_{1}} \mathrm{u}_{\left(\mathrm{s}_{1}\right)}\right)^{2}+\left(\frac{\partial \mathrm{g}}{\partial \mathrm{t}_{2}} \mathrm{u}_{\left(\mathrm{t}_{2}\right)}\right)^{2}+\left(\frac{\partial \mathrm{g}}{\partial \mathrm{t}_{1}} \mathrm{u}_{\left(\mathrm{t}_{1}\right)}\right)^{2}\right\} / \mathrm{g}^{2}
$$

In this experimental design, the uncertainty of Class B were used for all. In the measurement of the distance and time, the same measurement instrument were used, take $u_{\left(t_{1}\right)}=u_{\left(t_{2}\right)}=u_{t}$, $\mathrm{u}_{\left(\mathrm{s}_{1}\right)}=\mathrm{u}_{\left(\mathrm{s}_{2}\right)}=\mathrm{u}_{\mathrm{s}}$, we can get based on formula (12)

$$
s_{2} t_{1}-s_{1} t_{2}=1 / 2 g t_{1} t_{2}\left(t_{2}-t_{1}\right)
$$

$$
\left[\frac{\mathrm{u}(\mathrm{g})}{\mathrm{g}}\right]^{2}=\frac{1}{\left(\mathrm{t}_{2}-\mathrm{t}_{1}\right)^{2}}\left\{\left[\left(1+\frac{\mathrm{v}_{0}}{\mathrm{gt}_{1}}\right)^{2}+\left(1+\frac{\mathrm{v}_{0}}{\mathrm{gt}_{2}}\right)^{2}\right] \mathrm{u}_{\mathrm{t}}^{2}+\left[\left(\frac{1}{\mathrm{gt}_{1}}\right)^{2}+\left(1+\frac{1}{\mathrm{gt}_{2}}\right)^{2}\right] \mathrm{u}_{\mathrm{s}}{ }^{2}\right\}
$$

According to this formula, in order to minimize the value of $\left[\frac{\mathrm{u}(\mathrm{g})}{\mathrm{g}}\right]^{2}$, there are three cases:

First, when $s_{1}$ and $s_{2}$ take a certain value, $V_{0}$ take the minimum, that is, the value of $V_{0}$ tends to zero. Therefore, when the experiment is carried out, the position of $\mathrm{E}_{0}$ can be fixed at the place where the ball is originally dropped.

Second, when $t_{1}$ and $s_{1}$ take a certain value, value $t_{2}$ and $s_{2}$ taken is much larger, that is, try to fix E2 position at the bottom.

Thirdly, when the values of $t_{1}$ and $t_{2}$ are the most appropriate, the values of $s_{1}$ and $s_{2}$ are also optimal, and the following two formulas can be obtained by formula (16):

$$
\begin{aligned}
& \frac{\mathrm{d}}{\mathrm{dt}_{1}}\left\{\frac{1}{\left(\mathrm{t}_{2}-\mathrm{t}_{1}\right)^{2}}\left[\left(1+\frac{\mathrm{v}_{0}}{\mathrm{gt}_{1}}\right)^{2}+\left(1+\frac{\mathrm{v}_{0}}{\mathrm{gt}_{2}}\right)^{2}\right]\right\}=0 \\
& \frac{\mathrm{d}}{\mathrm{dt}_{1}}\left\{\frac{1}{\left(\mathrm{t}_{2}-\mathrm{t}_{1}\right)^{2}}\left[\left(\frac{1}{\mathrm{gt}_{1}}\right)^{2}+\left(\frac{1}{\mathrm{gt}_{2}}\right)^{2}\right]\right\}=0
\end{aligned}
$$

According to (12)(17) (18) these three formulas, we can eventually get the ratio of $s_{1}$ to $s_{2}$ is about 1 to 4 . 


\section{Analysis and discussion}

In the theory of uncertainty, the final measurement result is obtained by measuring the result of the most close to the true value of the object and the uncertainty of the measured value. Since the ultimate purpose of the measurement is to obtain the measured value and to determine whether the measurement is true and reliable, whether the measured results can be judged as measurement basis or not is on the basis of the uncertainty of the results. In general, when evaluating the quality of the measurement, the absolute and relative two uncertainties are used. If the final result shows that the overall uncertainty is greater than the value of the uncertainty measured by the instrument, but it is not significant, then we can assume that the measurement result can be used as a true and reliable basis because it is basically consistent with the measured value of the instrument. In the measurement, the purpose of the various methods of comparative analysis is to make the uncertainty can be smaller. In general, if the difference between the final result of the measurement and its true value is considered to be substantially uniform in the range of three times of the standard degree, we can believe the error range is consistent. This paper mainly through the minimum uncertainty to carry out experimental theory and measurement options, from the theoretical point of view, it is the further optimization to its previous experimental program, if applied properly, it can be applied to practice. The actual case combined with in the selection of experimental theory is the volume of the cylinder. By calculating it, we can see that when the specific conditions are different, it is necessary to ensure that the uncertain value can be minimized, and different methods should be applied in the calculation, That is, through its selection of the optimal experimental theory. In order to minimize the value of uncertainty, it is necessary to compare different measurement schemes and finally select the optimal measurement scheme.

\section{Conclusion}

In short, the accuracy of the measurement results are critical throughout the experiment, but it is common that more or less errors will appear in the measurement process, so how to control this error in the minimum range has become a very important issue. This paper is based on the theory of uncertainty, through the minimum uncertainty value to carry out experimental theory and program choice, has a certain theoretical significance.

\section{References}

[1] GaoYun-feng, Lan Feng, Qi Jingang. Comparison of experimental uncertainty and measurement error [J]. Journal of Changchun Normal University: Natural Science Edition, 2012 (10): 144-146.

[2] Wu Xiao, Zhou Shucang, Zhou Yuchang. Exploration of design experiment in college physics experiment [J]. Journal of Guangdong University of Technology, 2013 (6): 241-242.

[3] Wang Xiangchuan. Key grasp and application of error and uncertainty in the physical experiment teaching [J]. Journal of Chengdu University: Natural Science Edition, 2012 (3): 81-84.

[4] International Bureau of Metrology, International Electrotechnical Commission, International Organization for Standardization. Basic terminology of international general metrology [M]. Beijing: China Metrology Publishing House, 2014: 41-42.

[5] Wu Yuanxi, Xie Qing, Zhang Xiaoyu. The countermeasures to improve the level of undergraduate research experiment [J]. Laboratory Research and Exploration, 2012 (5): 31-34. 